\title{
Quantum features in the orthogonality of optical modes for structured and plane-wave light
}

\author{
David L. Andrews* (i) and Kayn A. Forbes (i) \\ School of Chemistry, University of East Anglia, Norwich NR4 7TJ, UK \\ *Corresponding author: david.andrews@physics.org
}

Received 11 May 2018; revised 8 June 2018; accepted 8 June 2018; posted 11 June 2018 (Doc. ID 331420$) ;$ published 5 July 2018

\begin{abstract}
A fundamental photon creation-annihilation commutation relation underpins the familiar quantum formulation of optics. However, an internal inconsistency becomes apparent in the pursuit of structured light applications. This requires the relationship between operator commutation and mode orthogonality to be recast in a form ensuring full consistency with the precepts of quantum theory. A suitable reformulation, shown to register correctly an intrinsic quantum uncertainty in the associated interactions, has special relevance to optical vortex physics-particularly with regard to information content-through its connection to the degrees of freedom in the associated radiation modes. () 2018 Optical Society of America
\end{abstract}

OCIS codes: (270.0270) Quantum optics; (270.5585) Quantum information and processing; (060.2605) Free-space optical communication; (080.4865) Optical vortices.

https://doi.org/10.1364/OL.43.003249

In the field of quantum optics, much of the basic theory is conventionally cast in terms that are primarily designed to apply to a single mode of radiation- a single wavelength, direction, and polarization. In the most obvious extension of these principles-allowing for more numerous modes of radiationthere are usually significant intervals between the accommodated frequencies, as, e.g., in the case of frequency combs [1], or else there are substantial differences in the directions of propagation. At the heart of most of the quantum formalism, there is a simple and widely familiar boson commutation relation between photon creation and annihilation operators [2]. One physical interpretation is that a photon propagating in a given radiation mode in vacuum cannot spontaneously divert into another mode.

In the sphere of optics, the creation-annihilation commutation relation is often presented in a mathematically concise form, neglecting polarization features that ought also, for generality, to be accommodated. Although these features are not commonly given attention, they supplement the wave vector information in uniquely defining each radiation mode. The commutation relation is specifically cast in terms of a binary basis, for although polarization measurements can be made at a spatially localized location with high fidelity, it is well understood that two linear polarization states whose electric vectors differ by only a few degrees cannot be regarded as orthogonal. However, for the directions of propagation associated with the wave vector, the assumption of an orthonormal basis is not so straightforward. Moreover the wave vector of light does not commonly engage with detection apparatusunless weak quadrupole attributes are engaged, which is rarely the case.

The difficulties of assuming an infinitely sharp distinction between modes of similar wave vectors are thrown into sharp relief in the case of structured radiation [3], in which there can be local variation of wave vector direction within the confines of a single well-defined beam. An optical vortex, or "twisted light," constitutes a prime example, where the wave vector represents the normal to a wavefront of helicoidal form. Significantly, it has been shown that the twisted character extends down to the level of individual photons [4,5], such that any given photon in a structured beam may manifest a variation in wave vector direction within the plane that is transverse to the axis of propagation. Part of the problem is that the nominal quantization volume, within which distinct photons are numbered, is invariably assumed to be substantially larger than an optical wavelength-and it is accordingly a volume that contains a markedly non-singular distribution of wave vectors.

It emerges that the conventional cast of the creationannihilation commutation relation, which imposes complete orthogonality between modes with infinitesimal differences in propagation direction, is not only incorrect, but also inconsistent with fundamental precepts of quantum theory. It should be understood that any ultimate imprecision in establishing a difference between photons of marginally different direction is not simply a reflection of technical, experimental, or other interaction-related obstacles. Rather, it has to be an intrinsic feature of the quantum foundations for light itself. In this Letter, we re-examine the key commutation relation, and show that a different formulation is entirely consistent with quantum principles. Moreover, the new formulation has special relevance for the propagation character, and potential informationconveying capacity, of structured light [6]. The motivations behind the study are to highlight the importance of a correct photon commutation relation, to explore a connection to beams endowed with orbital angular momentum (OAM) [7], 
and to indicate implications for the burgeoning field of informatics using singular optics.

Photons are the elementary quantum excitations of any electromagnetic field, each associated with a specific radiation mode conventionally defined by wave vector and polarization. Designating such modes with labels $m, m^{\prime}$, the boson character is exhibited through the canonical commutation relations for the photon annihilation operator $a_{m}$, and counterpart creation operator $a_{m^{\prime}}^{\dagger}$, written in Kronecker delta form:

$$
\left[a_{m} a_{m^{\prime}}^{\dagger}\right]=\delta_{m m^{\prime}},
$$

which has been asserted as the starting point for the entirety of quantum optics [2]. In many applications, the above relation is interpreted only in terms of modes potentially differing in wave vector alone-either through measurably different wavelengths or directions of propagation. However, the above clearly lacks the detail to account for other conditions. Developing Eq. (1) in terms of the associated modal wave vectors $\boldsymbol{k}$ and $\boldsymbol{k}^{\prime}$, and polarization states denoted by labels $\eta$ and $\eta^{\prime}$ leads to an apparently more complete cast of the commutation relations [8]:

$$
\left[a^{(\eta)}(\boldsymbol{k}), a^{\dagger\left(\eta^{\prime}\right)}\left(\boldsymbol{k}^{\prime}\right)\right]=\delta_{\boldsymbol{k} \boldsymbol{k}^{\prime}} \delta_{\eta \eta^{\prime}}
$$

However, although the commutation relation (2) is more accurate than the more generalized form (1), both representations are flawed. The issue is more easily identified in the latter, whereby the first (Kronecker) delta clearly has an improper form, containing pairs of indices that do not form an orthogonal basis.

The incorrect commutation relation (2) can be recast, using a Dirac delta function (of which the Kronecker delta is a discrete analogue), into a form that is suitable for the limiting case of an infinite plane wave [9]:

$$
\begin{aligned}
& {\left[a^{(\eta)}(\boldsymbol{k}), a^{\dagger\left(\eta^{\prime}\right)}\left(\boldsymbol{k}^{\prime}\right)\right]} \\
& =\left(8 \pi^{3} V\right)^{-1} \delta^{3}\left(\boldsymbol{k}-\boldsymbol{k}^{\prime}\right) \delta_{\eta \eta^{\prime}} \\
& =\left(8 \pi^{3} V\right)^{-1} \delta\left(k_{x}-k_{x}^{\prime}\right) \delta\left(k_{y}-k_{y}^{\prime}\right) \delta\left(k_{z}-k_{z}^{\prime}\right) \delta_{\eta \eta^{\prime}},
\end{aligned}
$$

where $V$ is the quantization volume, and the single Kronecker delta in Eq. (3) is fully restricted to orthogonal basis sets-such as might be represented by any pair of points on opposite sides of a Poincaré sphere. This orthogonality of basis polarization states correlates with the usual binary basis for conveying information.

It is readily verified that the constants on the right-hand side of Eq. (3) satisfy the dimensionless character of the commutator. With regard to its $[L]^{3}$ dimensions, deployment of the Dirac delta necessarily relates to a three-dimensional region of space, here registered by the quantization volume $V$. With increasing $V$, there is an increasing capacity to distinguish between photons with wave vectors of marginally different angular disposition. It is noteworthy that each Dirac delta function can be expressed as any one the numerous limit representations [10], and those limits may in principle be considered as physically corresponding to a quantization volume of infinite size. This signifies that there are no boundary constraints on the wave vectors, which therefore constitute a continuous, unbounded set in three-dimensional space.

Before proceeding further, it is useful to consider the implications for physical environments, or forms of radiation, when these assumptions fail. If we restrict attention to two modes whose photons have a common frequency $\omega=c|\boldsymbol{k}|=c\left|\boldsymbol{k}^{\prime}\right|$, but marginally different directions of propagation (and therefore unequal vector momenta), then the non-zero commutator in Eq. (3) signifies a degree of compromise to the possibility of local discrimination between photons in these two modes. This conclusion, entirely consistent with position-momentum uncertainty, thus invites closer scrutiny for light with inbuilt variations in wave-vector directionality.

Having addressed the fundamental issues of the photon annihilation-creation commutation relation for plane waves, the archetype of propagating light, we may now follow similar principles for so-called "structured" or "complex" light. Having a mathematically correct and physically defensible form for the creation-annihilation commutation relations is clearly essential. For structured laser light with a specific direction of propagation, Eq. (3) is more suitably recast in cylindrical coordinates. As shown in Fig. 1, the three degrees of freedom for the wave-vector $\boldsymbol{k}$ are now developed as axial, radial, and azimuthal components: $k_{z}, k_{\rho}$, and $k_{\phi}$ (the angular dependence of the latter cast in wavenumber dimensions for consistency with the other two components):

$$
\begin{aligned}
{\left[a^{(\eta)}(\boldsymbol{k}), a^{\dagger\left(\eta^{\prime}\right)}\left(\boldsymbol{k}^{\prime}\right)\right]=} & \left(8 \pi^{3} V\right)^{-1} \delta\left(k_{z}-k_{z}^{\prime}\right) \\
& \times \delta\left(k_{\rho}-k_{\rho}^{\prime}\right) \delta\left(k_{\phi}-k_{\phi}^{\prime}\right) \delta_{\eta \eta^{\prime}} .
\end{aligned}
$$

In the limit of Eq. (4), the product of four delta functions appears to signify that if two radiation modes differ in any one or more of the parameters $k_{z}, k_{\rho}, k_{\varphi}$, and $\eta$, they are orthogonal — and therefore have the capacity to independently convey information. However, it will be shown that precise modal orthogonality cannot be secured-not at least through experimental limitations, but also for more fundamental quantum uncertainty reasons.

To highlight such quantum issues, we consider the most studied vortex form of structured light: Laguerre-Gaussian (LG) modes. In the quantum operator formulation, each register of the electromagnetic field (e.g., electric or magnetic field) is now cast as a sum over modes designated by four distinct parameters [11] $-k, \eta, \ell, p$, representing a partition into longitudinal, polarization, angular, and radial functions-thereby signifying four degrees of freedom for the photon. This contrasts with the usual four degrees of freedom in the conventional plane wave expansions, consisting of three Cartesian components of the wave vector and one for the polarization state. It is this disparity between plane-wave and structured

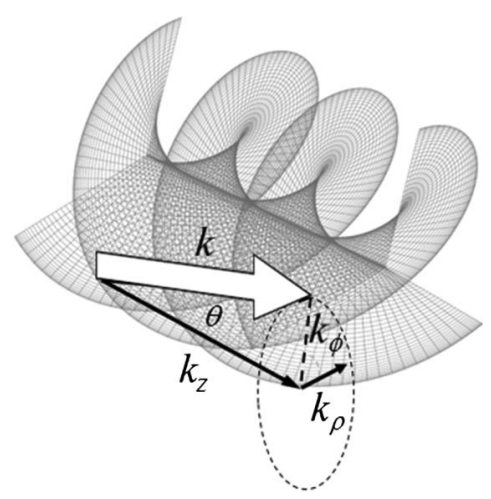

Fig. 1. Cylindrical vector components of the wave vector in a vortex beam. Note that the $k_{z}$ vector component need not be identified with the beam axis: this is a representation in reciprocal space. 
radiation, which forms the basis for the conveyance of additional information, density for a photon with the character of the latter form of light, i.e., a photon possessing OAM, can carry more information than its plane-wave counterpart $[12,13]$.

The orthogonality of disparate modes-through either their radial or angular functions, or both-offers a basis for determining the structural information content. The azimuthal functions in the field operators emerge in the form of simple phase factors $\mathrm{e}^{i \ell \phi}$, consistent with the orthogonality of modes with different topological charges. Most applications seeking to exploit mode orthogonality, for a given $k, \eta$, consider only the differing values of topological charge (winding number) $\ell$ : the index $p$ is either not accounted for, or simply assumed to be 0 . However, the radial index $p$ has to be included in the mode summation, for generality and completeness of the modal decomposition [14]. Indeed, there has been a recent surge in interest in the radial term, and it has been sought to substantiate the role $p$ can play in informatics [15-17]. Of course, $\ell$ and $p$ both feature in the detailed form of the LaguerreGaussian radial distribution:

$$
f_{\ell, p}(\rho)=\frac{C_{|\ell|}^{p}}{w_{0}}\left[\frac{\sqrt{2} \rho}{w_{0}}\right]^{|\ell|} \exp \left(\frac{-\rho^{2}}{w_{0}^{2}}\right) L_{|\ell|}^{p}\left(\frac{2 \rho^{2}}{w_{0}^{2}}\right),
$$

where $C_{|\ell|}^{p}$ is a normalization constant, and $w_{0}$ is the Gaussian beam waist at $z=0$. The above expression is cast as the familiar product of a Gaussian with an associated Laguerre polynomial $L_{\ell}^{p}(x)$, the latter being one of the solutions of the following partial differential equation:

$$
x \frac{\partial^{2} L_{\ell}^{p}(x)}{\partial x^{2}}+(p+1-x) \frac{\partial L_{\ell}^{p}(x)}{\partial x}+\ell L_{\ell}^{p}(x)=0 .
$$

Since $\ell$ appears in Eq. (5) in the guise of its modulus, vortices of opposite topological charge have identical radial distributions; their physical differences arise through their phase factors [18].

Orthogonality among the associated Laguerre polynomials is usually specified with respect to those of the same index $p$. The form that is relevant for Laguerre-Gaussian beam applications, which also introduces a weighting factor, is expressible as follows:

$$
\int_{0}^{\infty} e^{-x} x^{p} L_{\ell}^{p}(x) L_{\ell^{\prime}}^{p}(x) \mathrm{d} x=\frac{(\ell+p) !}{\ell !} \delta_{\ell \ell^{\prime}} .
$$

However, with $p \neq p^{\prime}$, an expression of the following general form arises, cast in terms of hypergeometric functions [19]:

$$
\begin{aligned}
& \int_{0}^{\infty} e^{-x} x^{\mu} L_{\ell}^{p}(x) L_{\ell^{\prime}}^{p^{\prime}}(x) \mathrm{d} x \\
& =\left(\begin{array}{c}
\ell+p \\
\ell
\end{array}\right)\left(\begin{array}{c}
\ell^{\prime}+p^{\prime}-\mu-1 \\
\ell^{\prime}
\end{array}\right) \\
& \quad \times{ }_{3} F_{2}\left(-\ell, \mu+1, \mu-p^{\prime}+1 ; p+1, \mu-p^{\prime}-\ell^{\prime}+1 ; 1\right) \\
& \quad \times \Gamma(\mu+1),
\end{aligned}
$$

which holds under the conditions that are satisfied in our application, i.e., integer $\ell, \ell^{\prime}$ and real $\mu, \Re(\mu)>-1$.

Returning to the commutation relations, the modal discriminators of radial and azimuthal form reduce to the familiar Kronecker form appropriate to a properly orthonormal basis set. Accordingly, the quantization volume is now cast in terms of a lateral extension $z$ along the propagation axis:

$$
\left[a_{\ell, p}^{(\eta)}(k \hat{z}), a_{\ell^{\prime}, p^{\prime}}^{\dagger\left(\eta^{\prime}\right)}\left(k^{\prime} \hat{z}\right)\right] \cong(2 \pi z)^{-1} \delta\left(k_{z}-k_{z}^{\prime}\right) \delta_{\ell \ell^{\prime}} \delta_{p p^{\prime}} \delta_{\eta \eta^{\prime}} .
$$

Although a result of this form emerges on integration over the nominally infinite extent of a plane transverse to the propagation axis $z$, its validity is contingent upon the fulfillment of the specific condition $p=p^{\prime}$ (and $p=p^{\prime}=0$ is frequently experimentally favored). In other circumstances, explorations of radial function discrimination may be compromised by the evaluations of Eq. (9), giving results that are no longer simply expressible in terms of the Kronecker delta $\delta_{p p^{\prime}}$-see Eq. (7).

We now focus on the physical significance of the first delta on the right of Eq. (9). To accommodate quantum uncertainty, it is appropriate to recast the longitudinal wave-vector constraint in terms of a limit. There are various choices available for representing the Dirac delta function in such a form. For our present purposes-departing from an earlier formulation [9], and arguably more true to the physicality of beam propagation - we choose the form of a sinc function, effectively representing the extent of local divergence between beams with axial wave-vector components $k_{z}$ and $k_{z}^{\prime}$ :

$$
\begin{aligned}
\delta\left(k_{z}-k_{z}^{\prime}\right) & =\lim _{\varepsilon \rightarrow 0}\left[\frac{\sin \left(k_{z}-k_{z}^{\prime}\right) / \varepsilon}{\pi\left(k_{z}-k_{z}^{\prime}\right)}\right] \\
& =\lim _{\varepsilon \rightarrow 0}(\pi \varepsilon)^{-1} \operatorname{sinc}\left[(\varepsilon z)^{-1}\left(\boldsymbol{k}-\boldsymbol{k}^{\prime}\right) \cdot \boldsymbol{z}\right] .
\end{aligned}
$$

The scalar multiplier $\varepsilon^{-1}$ in the even sinc function, in its limit, essentially compensates the effect on that function of increasing the range $z$. The sinc function is, of course, similar in form to the amplitude for forward scattering of a plane-wave electromagnetic field of wave-vector $\boldsymbol{k}$ at a position $z$, measured at a position $\boldsymbol{z}^{\prime}$-which is sinc $\boldsymbol{k} .\left(\boldsymbol{z}-\boldsymbol{z}^{\prime}\right)$, whose delta function limit in the far field ensures a conservation of linear momentum. Writing $\boldsymbol{k}^{\prime}=\boldsymbol{k}+\delta \boldsymbol{k}$ and $\boldsymbol{z}^{\prime}=\boldsymbol{z}+\delta \boldsymbol{z}$, and discarding $\delta^{2}$ terms, underscores the comparison. The connection has the physical significance that only by registering the electromagnetic field at two separate positions can the direction of a wave vector be determined: the same principle naturally applies in the case of a quadrupolar interaction, measured by an extended charge distribution. Once again, the physical implication revealed by expressing the Dirac delta on the left of the above equation as a limit of the function on the right is that in any regime short of the asymptote, the creation-annihilation commutator is not precisely zero in further correlation with the principle of position-momentum uncertainty.

We now focus on the local variation in wave-vector direction, which is intrinsic in the nature of structured light. It is especially instructive to examine further the implications of the reformulated delta function with a specific focus on optical vortices. To do so, it is necessary to correct a widely supposed relation between the topological charge and the pitch angle of the helicoidal wavefront, i.e., the pitch angle $\theta$ in Fig. 1 . Yeganeh et al. [20] and Bekshaev et al. [21], e.g., report the pitch angle as $\theta=\ell / k \rho$, but this cannot be correct, since it implies $\theta>\pi / 2$ for large enough $\ell$-which would signify a reversal of handedness. The correct form of the relationship has to be $\tan \theta=\ell / k \rho$, which correctly gives $\theta=0$ for $\ell=0$ and $\theta=\pi / 2$ for $\ell=\infty$. Moreover, the usual form of expression is consistent with the small angle limit of the tangent. Of course, the singular nature of the beam means there is zero intensity along the axis where $\rho=0$. The behavior of the argument for the sinc function in Eq. (10) can be pursued by 
assigning primed and unprimed locational coordinates to the positions for measurement of the correspondingly primed and unprimed modes. For two locations, $(z, \rho, \phi)$ and $(z, \rho+\delta \rho, \phi)$, which differ only marginally in radial position, we define a parameter $g$ as

$$
\begin{aligned}
g\left(\boldsymbol{k} ; z, \rho, \phi \mid \boldsymbol{k}^{\prime} ; z, \rho^{\prime}, \phi\right) \equiv & z^{-1}\left(\boldsymbol{k}-\boldsymbol{k}^{\prime}\right) \cdot \boldsymbol{z} \\
= & k\left(\cos \theta-\cos \theta^{\prime}\right) \\
= & k\left[\left(1+\ell^{2} /(k \rho)^{2}\right)^{-1 / 2}\right. \\
& \left.-\left(1+\ell^{2} /\left(k \rho^{\prime}\right)^{2}\right)^{-1 / 2}\right],
\end{aligned}
$$

and therefore,

$$
\begin{aligned}
\delta g & =-\frac{k}{2}\left[\left(1+\ell^{2} /(k \rho)^{2}\right)^{-3 / 2}(-2)\left(\ell^{2} /\left(k^{2} \rho^{3}\right)\right)\right] \delta \rho \\
& =\frac{\ell^{2}}{k \rho^{3}}\left(1+\ell^{2} /(k \rho)^{2}\right)^{-3 / 2} \delta \rho .
\end{aligned}
$$

For small $\rho$, and/or small $k$, and/or large $\ell$, signifying large $\theta$, the result approximates to

$$
\delta g \approx \frac{\ell^{2}}{k \rho^{3}}\left(\ell^{2} /(k \rho)^{2}\right)^{-3 / 2} \delta \rho=\frac{\ell^{2}}{k \rho^{3}} \frac{k^{3} \rho^{3}}{\ell^{3}} \delta \rho=\frac{k^{2}}{\ell} \delta \rho .
$$

On the other hand, for large $\rho$, and/or large $k$, and/or small $\ell$, signifying small $\theta$, we have the asymptotic form:

$$
\delta g \approx \frac{\ell^{2}}{k \rho^{3}} \delta \rho .
$$

The physical significance in the former case-such as for positions close to the longitudinal axis - is that an uncertainty in measurability of the angular distinction $\delta \theta$, between the associated locally oriented wave vectors, is inversely proportional to $\ell$. Since the OAM per photons is $\ell \hbar$, this is entirely consistent with the angle-angular momentum uncertainty principle for structured light [22]. The latter asymptote, which might equally be conceived for positions remote from the axis, signifies that the measurability of the differential $\delta \theta$ scales quadratically with $\ell$, but there is a much more demanding limit on the degree of non-collinearity that can escape detection.

Methods that have been devised to sort optical modes on the basis of their OAM have proven that each mode can confidently be sorted from a range of 10 or more other, numerically adjacent $\ell$ values. Nonetheless, incomplete differentiation is evident in the results of most experiments [23,24]. Imperfections are also apparent in groundbreaking experiments on the multiplication of OAM values [25]. Some of these imperfections could certainly arise from technical limitations; however, our conclusion is that at the limits of experimental resolution-or below it - there is a fundamental limit on modal orthogonality, of quantum character, which cannot be overcome. Since it has also been shown that individual photons register mode topology [26], the true extent of associated information conveyance per photon presents an issue of technical relevance to schemes for the high-fidelity encoding and sorting of information using structured light. By tackling the fundamental photon physics, our work has aimed to address and advance the underlying quantum theory. Possible implications for the propagation of guided modes now invite further scrutiny.

\section{REFERENCES}

1. C. Reimer, M. Kues, P. Roztocki, B. Wetzel, F. Grazioso, B. E. Little, S. T. Chu, T. Johnston, Y. Bromberg, L. Caspani, D. J. Moss, and R. Morandotti, Science 351, 1176 (2016).

2. G. Grynberg, A. Aspect, and C. Fabre, Introduction to Quantum Optics: From the Semi-Classical Approach to Quantized Light (Cambridge University, 2010).

3. D. L. Andrews, ed., Structured Light and Its Applications: An Introduction to Phase-Structured Beams and Nanoscale Optical Forces (Academic, 2008).

4. M. Krenn, M. Malik, M. Erhard, and A. Zeilinger, Philos. Trans. R. Soc. A 375, 20150442 (2017).

5. J. Leach, M. J. Padgett, S. M. Barnett, S. Franke-Arnold, and J. Courtial, Phys. Rev. Lett. 88, 257901 (2002).

6. G. Xie, L. Li, Y. Ren, H. Huang, Y. Yan, N. Ahmed, Z. Zhao, M. P. J. Lavery, N. Ashrafi, S. Ashrafi, R. Bock, M. Tur, A. F. Molisch, and A. E. Willner, Optica 2, 357 (2015).

7. M. Erhard, R. Fickler, M. Krenn, and A. Zeilinger, Light Sci. Appl. 7, 17146 (2018).

8. R. Loudon, The Quantum Theory of Light (Oxford University, 2000).

9. M. D. Williams, D. S. Bradshaw, and D. L. Andrews, Proc. SPIE 9764, 976407 (2016).

10. K. F. Riley, M. P. Hobson, and S. J. Bence, Mathematical Methods for Physics and Engineering: A Comprehensive Guide (Cambridge University, 2006).

11. L. C. Dávila Romero, D. L. Andrews, and M. Babiker, J. Opt. B 4, S66 (2002).

12. G. Gibson, J. Courtial, M. J. Padgett, M. Vasnetsov, V. Pas'ko, S. M. Barnett, and S. Franke-Arnold, Opt. Express 12, 5448 (2004).

13. J. Wang, J.-Y. Yang, I. M. Fazal, N. Ahmed, Y. Yan, H. Huang, Y. Ren, Y. Yue, S. Dolinar, M. Tur, and A. E. Willner, Nat. Photonics 6, 488 (2012).

14. C. Schulze, A. Dudley, D. Flamm, M. Duparré, and A. Forbes, New J. Phys. 15, 073025 (2013).

15. E. Karimi, R. W. Boyd, P. de la Hoz, H. de Guise, J. Řeháček, Z Hradil, A. Aiello, G. Leuchs, and L. L. Sánchez-Soto, Phys. Rev. A 89, 063813 (2014).

16. W. N. Plick and M. Krenn, Phys. Rev. A 92, 063841 (2015).

17. Y. Zhou, M. Mirhosseini, D. Fu, J. Zhao, S. M. H. Rafsanjani, A. E. Willner, and R. W. Boyd, Phys. Rev. Lett. 119, 263602 (2017).

18. K. A. Forbes and D. L. Andrews, Opt. Lett. 43, 435 (2018).

19. H. A. Mavromatis, Int. J. Comput. Math. 36, 257 (1990).

20. M. Yeganeh, S. Rasouli, M. Dashti, S. Slussarenko, E. Santamato, and E. Karimi, Opt. Lett. 38, 887 (2013).

21. A. Y. Bekshaev, I. Kurka, K. Mohammed, and I. Slobodeniuk, Ukr. J. Phys. Opt. 16, 17 (2015).

22. S. Franke-Arnold, S. M. Barnett, E. Yao, J. Leach, J. Courtial, and M. Padgett, New J. Phys. 6, 103 (2004).

23. G. C. Berkhout, M. P. Lavery, J. Courtial, M. W. Beijersbergen, and M. J. Padgett, Phys. Rev. Lett. 105, 153601 (2010).

24. M. Mirhosseini, O. S. Magaña-Loaiza, M. N. O'sullivan, B. Rodenburg, M. Malik, M. P. J. Lavery, M. J. Padgett, D. J. Gauthier, and R. W. Boyd, New J. Phys. 17, 033033 (2015)

25. V. Potoček, F. M. Miatto, M. Mirhosseini, O. S. Magaña-Loaiza, A. C. Liapis, D. K. L. Oi, R. W. Boyd, and J. Jeffers, Phys. Rev. Lett. 115, 160505 (2015).

26. E. J. Galvez, L. E. Coyle, E. Johnson, and B. J. Reschovsky, New J. Phys. 13, 053017 (2011) 